\title{
SELF-ESTEEM, QUALITY OF LIFE, AND PHYSICAL ACTIVITY IN WOMEN WITH DIAGNOSED ENDOMETRIOSIS: A PILOT STUDY IN AN ITALIAN SAMPLE
}

original paper

( ) University School of Physical Education in Wroclaw

DOI: https://doi.org/10.5114/hm.2021.103295

\section{ANTONINO BIANCO ${ }^{1}$, AMBRA GENTILE ${ }^{1}$, MARZIA RUSSO ${ }^{1}$, ANTONIO MAIORANA ${ }^{2}$, GIOACCHINO LAVANCO $^{1}$, ARIANNA MELLINA ${ }^{2}$, ANTONIO PALMA ${ }^{1}$}

${ }^{1}$ Department of Psychology, Educational Science and Human Movement, University of Palermo, Palermo, Italy

${ }^{2}$ Gynaecologic and Obstetric Unit, ARNAS Civico Di Cristina Fatebenefratelli, Palermo, Italy

\begin{abstract}
Purpose. Endometriosis is a painful pathology impairing women's daily life. Several studies have shown that patients report low mental and physical quality of life, but no general evaluation of their self-esteem has been found. Studies concerning physical activity frequency present inconsistent results. Therefore, the current paper aims to compare life quality and selfesteem in women with endometriosis with the Italian normative data of the measures used.

Methods. Overall, 36 women with diagnosed endometriosis treated in hospital completed a questionnaire detecting physical activity frequency, self-esteem, quality of life, and socioeconomic information. One sample $t$-test served to compare the data from this particular group with those of the general Italian population.

Results. The participants displayed higher self-esteem $(t=5.65, p<0.001)$ and a good quality of life (physical functioning: $t=3.64, p<0.001)$, except for fatigue $(t=-3.24, p=0.003$; significant difference); they declared feeling more fatigue than the general population. Only 4 women (11\%) reported regular physical activity, while 16 were smokers $(44.4 \%$, mean: 10.6 cigarettes/day).

Conclusions. Women with diagnosed endometriosis presented higher self-esteem and normal quality of life, even though their general health was lower and fatigue was higher than in the general Italian population. However, the sample involved females of low socioeconomic status and low education, characterized by poor communication skills and not open to externalize their feelings. Therefore, they might not have been honest in their answers. This potential bias, together with the small sample size, will be addressed in future research.
\end{abstract}

Key words: endometriosis, self-esteem, quality of life, dyspareunia, pain

\section{Introduction}

Endometriosis is a pathological condition where endometrial tissue that normally grows inside the uterus is also found outside the uterus, causing chronic pelvic pain [1]. Women with endometriosis tend to experience painful periods, chronic lower abdominal pain, infertility, and dyspareunia [2]. Since the diagnosis of endometriosis is often based on reported symptoms, as the occurrence of pain, its identification is characterized by a delay of almost 10 years [3], leaving women in a depressed or frustrated mood [4]. Therefore, women with diagnosed endometriosis may experience problems in their social, sexual, and emotional daily life. A meta-analysis performed by Jia et al. [5] reports a significant impairment of women's healthrelated quality of life, finding a negative correlation with pelvic pain intensity.

The negative impact of this pathology on quality of life may turn into impairment of self-esteem since the symptoms experienced may limit women's daily activity, with a sense of general dissatisfaction concerning the self, described as a collection of attitudes towards one's personal characteristics and potentialities [6]. Some studies described low self-esteem as a consequence of infertility and in relation to motherhood [7], to dyspareunia, and to lack of sexual relationship [8]. Only one study relating self-esteem to the

Correspondence address: Antonino Bianco, University of Palermo, Via Pascoli, 6, 90144, Palermo, Italy, e-mail: antonino.bianco@unipa.it

Received: June 9, 2020

Accepted for publication: September 6, 2020

Citation: Bianco A, Gentile A, Russo M, Maiorana A, Lavanco G, Mellina A, Palma A. Self-esteem, quality of life, and physical activity in women with diagnosed endometriosis: a pilot study in an Italian sample. Hum Mov. 2021;22(4):93-97; doi: https://doi.org/10.5114/hm.2021.103295. 
experience of pain was found [9], reporting a non-significant correlation.

Finally, as presented by Bonocher et al. [10], physical activity has been used to reduce painful symptoms in diseases involving inflammatory processes. Therefore, the authors reviewed the literature for assessing the relationship between physical exercise and the prevalence and/or improvement of the symptoms associated with endometriosis. Unfortunately, owing to the small number of studies existing on endometriosis, these results were inconsistent. Conversely, stretching activities, as yoga, seem to have beneficial effects on quality of life and chronic pelvic pain [11].

Even though quality of life of women with diagnosed endometriosis has been already investigated, there is a lack of studies on a general evaluation of selfesteem and endometriosis. The retrieved studies associated endometriosis with specific symptoms or conditions (pain, infertility, dyspareunia, distress), but since it can manifest differently from woman to woman, a general evaluation of self-esteem could be useful to understand if there is a common feature in women who are affected by this pathology. Thus, the current study aimed at evaluating self-esteem, quality of life, smoking and alcohol habits, and frequency of physical activity in a sample of women with diagnosed endometriosis. Specifically, we expected that women with diagnosed endometriosis would experience a lower quality of life and lower self-esteem than the Italian population.

\section{Material and methods}

\section{Participants}

Data were collected at the gynaecologic unit of the ARNAS Civico of Palermo hospital. All women who received a diagnosis of endometriosis in the hospital unit underwent assessment at a medical visit. The sample was composed of 36 women aged 19-53 years (mean age: $36.97, S D=8.22$ ). The information was collected during a face-to-face interview at the hospital unit. The Italian personal data protection code (DL196/2003) was applied.

\section{Measures}

\section{Rosenberg Self-Esteem Scale}

Self-esteem was investigated with the Rosenberg Self-Esteem Scale [12], a questionnaire consisting of 10 items, each with a 4-point Likert scale (from $1=$
'Totally disagree' to 4 = 'Totally agree'), for a maximum total score of 40 points. The Italian standardization provides a good reliability of the measure and indicates normative data depending on gender, educational level, marital status, and occupation. For the purpose of the study, the normative data for Italian women derived from the standardization were used as a reference category.

\section{Short Form (36) Health Survey}

Health-related quality of life was measured with the Short Form (36) Health Survey (SF-36) [13], which consists of 36 questions divided into 8 scales relating to: physical functioning (10 items), role limitation owing to physical health (4 items), role limitation owing to emotional problems (3 items), vitality (4 items), emotional well-being (5 items), social functioning ( 2 items), bodily pain ( 2 items), and general health (5 items). The Italian standardization of the questionnaire was used, and the scores were calculated with the procedure suggested by Ware and Sherbourne [14].

\section{Questionnaire on sociodemographic data, lifestyle, and habits}

A questionnaire concerning sociodemographic data, lifestyle aspects, and habits was administered. It consisted of a sociodemographic part regarding age, height, weight, educational level. The other part included questions related to sleeping hours per night ('How many hours do you sleep per night?'), smoking habits ('Do you smoke?' with possible answers: Yes/No; if yes, 'How many cigarettes do you smoke per day?'), physical activity ('How many hours of physical activity do you practice weekly?'), and alcohol consumption ('Do you drink alcohol?'; if yes, 'How many glasses of alcoholic drinks do you consume weekly?').

\section{Data analysis}

Descriptive statistics were calculated for all the variables involved in this study. One sample $t$-test was used to compare the scores detected through our measures with the normative data for the population $[12,13]$. The significance level was set at $\alpha=0.05$. The Statistica software (version 10 for Windows) was applied.

\section{Ethical approval}

The research related to human use has complied with all the relevant national regulations and institu- 
tional policies, has followed the tenets of the Declaration of Helsinki, and has been approved by the Sport and Exercise Science Research Unit of the University of Palermo.

\section{Informed consent}

Informed consent has been obtained from all individuals included in this study.

\section{Results}

Descriptive statistics are presented in Table 1. The participants belonged to normal weight category of body mass index (mean: 23.7, $S D=3.66$ ) and had low to medium education degree (only 2 individuals had university graduation). The average number of sleeping hours was 6.68 hours/night $(S D=1.54)$. Only 4 women (11.1\%) declared to dedicate some time to physical activity, with an average of 5.33 hours per week $(S D=3.21)$. Out of the 36 patients, $16(44.4 \%)$ regularly smoked cigarettes, with an average of 10.6 cigarettes per day $(S D=6.07)$. Concerning alcohol consumption, 31 women (86.1\%) declared not to consume alcohol regularly, while 5 women (13.9\%) reported that they drank 1 glass of alcoholic beverages per week.

The comparison with the normative scores for the population returned non-significant differences concerning the following SF-36 scales: role limitations owing to physical health, bodily pain, general health, social functioning, role limitation owing to emotional problems, and emotional well-being. Significant differences were found with reference to vitality, where the women obtained lower scores compared with the normative data for the population $(t=-3.24, p=0.003)$, and physical functioning $(t=3.64, p<0.001)$. Self-esteem levels were higher in our sample than the results for the general population of women with low-to-medium educational level $(t=5.65, p<0.001)$. Although the scores did not reach the significance, it should be noted that women reported lower general health.

Table 1. Descriptive statistics of the sample and comparison with normative data

\begin{tabular}{|c|c|c|c|c|c|}
\hline Demographic characteristics & Mean (SD) & & & & \\
\hline Age (years) & $36.97(8.22)$ & & & & \\
\hline Height (cm) & $164.70(6.47)$ & & & & \\
\hline Weight (kg) & $64.29(11.27)$ & & & & \\
\hline Sleeping hours/night & $6.68(1.54)$ & & & & \\
\hline Education & Frequency & Percentage & & & \\
\hline Elementary school & 5 & $13.9 \%$ & & & \\
\hline Middle school (10-13 years) & 16 & $44.4 \%$ & & & \\
\hline High school & 13 & $36.1 \%$ & & & \\
\hline University & 2 & $5.6 \%$ & & & \\
\hline Lifestyle habits & Frequency & Percentage & & & \\
\hline Smoking (yes) & 16 & $44.4 \%$ & & & \\
\hline Alcohol consumption (yes) & 5 & $13.9 \%$ & & & \\
\hline Sport practice (yes) & 4 & $11.1 \%$ & & & \\
\hline SF-36 scales & $\begin{array}{c}\text { Sample } \\
\text { mean (SD) }\end{array}$ & $\begin{array}{c}\text { Normative data } \\
\text { mean (SD) }\end{array}$ & $\begin{array}{c}\text { Mean } \\
\text { difference }\end{array}$ & $t$ & $p$ \\
\hline Physical functioning & $91.67(11.89)$ & $84.46(23.18)$ & 7.21 & 3.64 & $<0.001 * *$ \\
\hline Role limitation owing to physical health & $84.29(35.92)$ & $78.21(35.93)$ & 6.08 & 1.00 & Non-significant \\
\hline Role limitation owing to emotional problems & $84.31(35.99)$ & $76.16(37.25)$ & 8.35 & 1.32 & Non-significant \\
\hline Vitality (energy/fatigue) & $52.22(17.90)$ & $61.89(20.69)$ & -9.67 & -3.24 & $0.003 *$ \\
\hline Emotional well-being & $60.56(20.06)$ & $66.59(20.89)$ & -6.03 & -1.80 & Non-significant \\
\hline Social functioning & $84.02(27.81)$ & $77.43(23.34)$ & 6.60 & 1.42 & Non-significant \\
\hline Bodily pain & $73.89(24.11)$ & $73.67(27.65)$ & 0.22 & 0.05 & Non-significant \\
\hline General health & $59.71(30.92)$ & $65.22(22.18)$ & -5.51 & -1.05 & Non-significant \\
\hline Self-esteem & $25.86(5.20)$ & $\begin{array}{l}30.89 \\
\text { (not reported) }\end{array}$ & 4.97 & 5.65 & $<0.001 * *$ \\
\hline
\end{tabular}

${ }^{*} p<0.05,{ }^{* *} p<0.001$ 


\section{Discussion}

The current study aimed to compare self-esteem and quality of life dimensions of women with diagnosed endometriosis with normative data obtained for the general population. Moreover, we wanted to detect the patients' lifestyle characteristics, particularly referring to physical activity. Our sample consisted of 36 women treated in the gynaecologic unit of the Civico Hospital, one of the main hospitals in Palermo.

The results contradicted our main hypothesis of lower self-esteem in women with diagnosed endometriosis, and partially contradicted also the one related to a lower quality of life. In fact, our sample presented high levels of self-esteem and a normal quality of life, except for the scale of vitality, where the results turned out lower than the normative scores. Also, women reported lower general health compared with the general population, even if this difference did not reach statistical significance. As for the patients' habits, the group was characterized by a high presence of smokers, with an average of more than 10 cigarettes per day; only a few individuals practised sports.

With regard to self-esteem, a few studies treating self-esteem as a predictor or consequence of specific endometriosis symptoms have been retrieved $[7-9,15]$. Conversely, we aimed to obtain a general evaluation of self-esteem in women affected by endometriosis, independently from the experienced symptoms. Our study found a high self-esteem level among the 36 women with endometriosis. Moreover, the severity of the symptoms should also impact on women's quality of life $[1,4]$. In our study, we observed a lack of energy in women with diagnosed endometriosis, but they reported normal levels, sometimes higher, in the other dimensions of quality of life.

These results should be interpreted under a cultural light: data were collected in a hospital situated in a poor area of the city, were people present a low socioeconomic status and low education level (21 participants went to school until the age of 13 years, while 13 gained a high school diploma). Since these individuals were characterized by scarce communicating skills, it is very likely that our results could have been biased for this cultural issue. Even if some women experienced pain or felt to be less capable than the others, the tendency was to suppress these thoughts and to rate oneself as better than the others.

The aim of the current research was to gain information useful for planning a future study about women with diagnosed endometriosis, their self-esteem, quality of life, and physical activity. For this reason, some limitations should be considered for the next phases. First of all, a bigger sample will be recruited by contacting participants from different Italian cities. It is more likely that employing a bigger sample, with heterogeneous characteristics concerning age and education, could lead to different results. Additionally, more information about the diagnosis of endometriosis should be collected, e.g. when it was detected, when the symptoms started, and what diagnostic methodology was used. Moreover, we plan to obtain more detailed information on pain intensity and pain localization, as well as to establish if this pain relates to an impairment in physical tasks. A section with more specific questions on physical activity will be included. Furthermore, as previously said, to overcome the cultural bias, the sample should be representative in terms of education and socioeconomic status, thus we intend to control these characteristics.

\section{Conclusions}

Women with diagnosed endometriosis were characterized by high self-esteem and normal quality of life. However, their general health was lower and fatigue was higher than in the general Italian population.

\section{Disclosure statement}

No author has any financial interest or received any financial benefit from this research.

\section{Conflict of interest}

The authors state no conflict of interest.

\section{References}

1. Lövkvist L, Boström P, Edlund M, Olovsson M. Age-related differences in quality of life in Swedish women with endometriosis. J Womens Health. 2016;25(6): 646-653; doi: 10.1089/jwh.2015.5403.

2. Fauconnier A, Fritel X, Chapron C. Endometriosis and pelvic pain: epidemiological evidence of the relationship and implications [in French]. Gynecol Obstet Fertil. 2009;37(1):57-69; doi: 10.1016/j.gyobfe.2008.08.016.

3. Hadfield R, Mardon H, Barlow D, Kennedy S. Delay in the diagnosis of endometriosis: a survey of women from the USA and the UK. Hum Reprod. 1996;11(4): 878-880; doi: 10.1093/oxfordjournals.humrep.a019270.

4. Sepulcri RdP, do Amaral VF. Depressive symptoms, anxiety, and quality of life in women with pelvic endometriosis. Eur J Obstet Gynecol Reprod Biol. 2009; 142(1):53-56; doi: 10.1016/j.ejogrb.2008.09.003.

5. Jia S-Z, Leng J-H, Shi J-H, Sun P-R, Lang J-H. Healthrelated quality of life in women with endometriosis: a systematic review. J Ovarian Res. 2012;5(1):29; doi: 10.1186/1757-2215-5-29. 
6. Juth V, Smyth JM, Santuzzi AM. How do you feel? Selfesteem predicts affect, stress, social interaction, and symptom severity during daily life in patients with chronic illness. J Health Psychol. 2008;13(7):884894; doi: 10.1177/1359105308095062.

7. Facchin F, Buggio L, Dridi D, Vercellini P. A woman's worth: the psychological impact of beliefs about motherhood, female identity, and infertility on childless women with endometriosis. J Health Psychol. 2019; 1359105319863093; doi: 10.1177/1359105319863093.

8. Denny E, Mann CH. Endometriosis-associated dyspareunia: the impact on women's lives. J Fam Plann Reprod Health Care. 2007;33(3):189-193; doi: 10.1783/ 147118907781004831.

9. Christian A. The relationship between women's symptoms of endometriosis and self-esteem. J Obstet Gynecol Neonatal Nurs. 1993;22(4):370-376; doi: 10.1111/ j.1552-6909.1993.tb01817.x.

10. Bonocher CM, Montenegro ML, Silva JCRE, Ferriani RA, Meola J. Endometriosis and physical exercises: a systematic review. Reprod Biol Endocrinol. 2014;12(1):4; doi: 10.1186/1477-7827-12-4.

11. Gonçalves AV, Barros NF, Bahamondes L. The practice of hatha yoga for the treatment of pain associated with endometriosis. J Altern Complement Med. 2017;23(1): 45-52; doi: 10.1089/acm.2015.0343.

12. Prezza M, Trombaccia FR, Armento L. The Rosenberg Self-Esteem Scale: Italian translation and validation [in Italian]. Giunti Organizzazioni Speciali. 1997;223: 35-44.

13. Apolone G, Mosconi P. The Italian SF-36 health survey: translation, validation and norming. J Clin Epidemiol. 1998;51(11):1025-1036; doi: 10.1016/s0895-4356(98) 00094-8.

14. Ware JE Jr, Sherbourne CD. The MOS 36-item shortform health survey (SF-36): I. Conceptual framework and item selection. Med Care. 1992;30(6):473-483.

15. Facchin F, Barbara G, Dridi D, Alberico D, Buggio L, Somigliana E, et al. Mental health in women with endometriosis: searching for predictors of psychological distress. Hum Reprod. 2017;32(9):1855-1861; doi: 10.1093/humrep/dex249. 